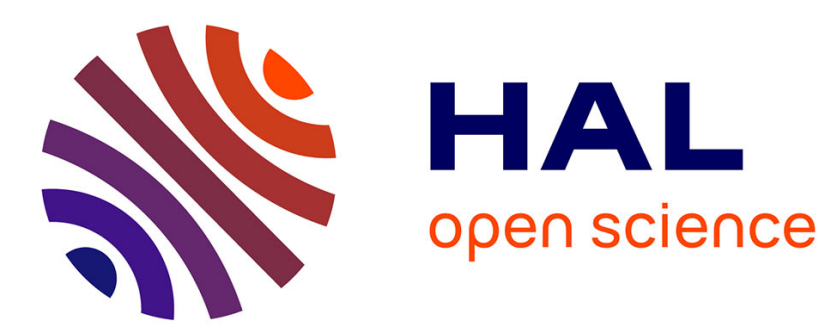

\title{
The motion of an axisymmetric body falling in a tube at moderate Reynolds numbers
}

Nicolas Brosse, Patricia Ern

\section{To cite this version:}

Nicolas Brosse, Patricia Ern. The motion of an axisymmetric body falling in a tube at moderate Reynolds numbers. Journal of Fluid Mechanics, 2013, vol. 714, pp. 238-257. 10.1017/jfm.2012.475 . hal-00904710

\section{HAL Id: hal-00904710 https://hal.science/hal-00904710}

Submitted on 15 Nov 2013

HAL is a multi-disciplinary open access archive for the deposit and dissemination of scientific research documents, whether they are published or not. The documents may come from teaching and research institutions in France or abroad, or from public or private research centers.
L'archive ouverte pluridisciplinaire HAL, est destinée au dépôt et à la diffusion de documents scientifiques de niveau recherche, publiés ou non, émanant des établissements d'enseignement et de recherche français ou étrangers, des laboratoires publics ou privés. 


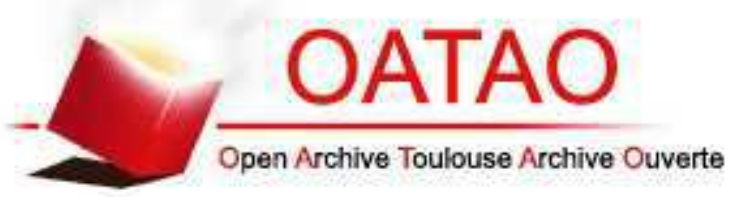

\section{Open Archive TOULOUSE Archive Ouverte (OATAO)}

OATAO is an open access repository that collects the work of Toulouse researchers and makes it freely available over the web where possible.

This is an author-deposited version published in : http://oatao.univ-toulouse.fr/ Eprints ID : 10098

To link to this article : DOI:10.1017/jfm.2012.475

URL : http://dx.doi.org/10.1017/jfm.2012.475

To cite this version : Brosse, Nicolas and Ern, Patricia. The motion of an axisymmetric body falling in a tube at moderate Reynolds numbers. (2013) Journal of Fluid Mechanics, vol. 714 . pp. 238-257. ISSN 0022-1120

Any correspondance concerning this service should be sent to the repository administrator: staff-oatao@ listes-diff.inp-toulouse.fr 


\title{
The motion of an axisymmetric body falling in a tube at moderate Reynolds numbers
}

\author{
Nicolas Brosse and Patricia Ern $\dagger$ \\ Université de Toulouse; INPT, UPS; Institut de Mécanique des Fluides de Toulouse; Allée Camille Soula, \\ F-31400 Toulouse, France \\ CNRS; Institut de Mécanique des Fluides de Toulouse; F-31400 Toulouse, France
}

This study concerns the rectilinear and periodic paths of an axisymmetric solid body (short-length cylinder and disk of diameter $d$ and thickness $h$ ) falling in a vertical tube of diameter $D$. We investigated experimentally the influence of the confinement ratio $(S=d / D<0.8)$ on the motion of the body, for different aspect ratios $(\chi=d / h=3$, 6 and 10), Reynolds numbers $(80<R e<320)$ and a density ratio between the fluid and the body close to unity. For a given body, the Reynolds number based on its mean vertical velocity is observed to decrease when $S$ increases. The critical Reynolds number for the onset of the periodic motion decreases with $S$ in the case of thin bodies $(\chi=10)$, whereas it appears unaffected by $S$ for thicker bodies $(\chi=3$ and 6$)$. The characteristics of the periodic motion are also strongly modified by the confinement ratio. A thick body $(\chi=3)$ tends to go back to a rectilinear path when $S$ increases, while a thin body $(\chi=10)$ displays oscillations of growing amplitude with $S$ until it touches the tube (at about $S=0.5$ ). For a given aspect ratio, however, the amplitudes of the oscillations follow a unique curve for all $S$, which depends only on the relative distance of the Reynolds number to the threshold of path instability. In parallel, numerical simulations of the wake of a body held fixed in a uniform confined flow were carried out. The simulations allowed us to determine in this configuration the effect of the confinement ratio on the thresholds for wake instability (loss of axial symmetry at $\operatorname{Re}_{c 1}$ and loss of stationarity at $\operatorname{Re}_{c 2}$ ) and on the maximal velocity $V_{w}$ in the recirculating region of the stationary axisymmetric wake. The evolution with $\chi$ and $S$ of $V_{w}$ at $R e_{c 1}$ was used to define a Reynolds number $R e^{*}$. Remarkably, for a freely moving body, $R e^{*}$ remains almost constant when $S$ varies, regardless of the nature of the path.

Key words: multiphase and particle-laden flows, vortex shedding, wakes/jets

\section{Introduction}

In the last decade, significant advance has been achieved concerning the understanding of the coupling between the motion of an isolated body freely rising/falling at moderate Reynolds numbers in an unbounded initially quiescent liquid and the perturbation the body induces in the liquid: see Ern et al. (2012) for a review. In particular, the relationship between the path of the body and the temporal and 
spatial characteristics of its wake, and their relationship to the instability of the wake of the body when fixed in an incoming flow, have been progressively clarified. This study is devoted to the effect of confinement on the body behaviour. The presence of the wall imposes an impermeability and no-slip constraint on the liquid motion induced by the moving body, which leads to the existence of a boundary layer at the wall. While both the intensity of the vorticity produced at the body surface and that of the wall boundary layer induced by the body motion depend on the Reynolds number $R e$, their relative strength and interaction depend on the distance of the body to the wall and on the level of confinement. The latter is characterized by the confinement ratio $S=d / D$, comparing a characteristic length $d$ of the body to the width $D$ of the tube or channel. As soon as the symmetry of the confinement is broken, e.g. a body falling off-centre in a tube, the wake structure is modified, resulting in asymmetric loads on the body, which induce its rotation and/or horizontal displacement. This is also observed in the semi-unbounded configuration of a body freely rising/falling along a plane wall. In this case potential flow approximation predicts wall attraction, since velocity (pressure) is higher (lower) in between the body and the wall. In contrast, the asymmetry imposed by the wall on the velocity field related to the production of vorticity at the body surface results in repulsion from the wall. The subtle balance between these two effects is discussed by Takemura \& Magnaudet (2003) in the case of contaminated and uncontaminated bubbles rising along a vertical wall, which experience attraction or repulsion from the wall depending on the Reynolds number and the distance to the wall. In the related situation of two fixed bodies placed side by side in an incoming flow investigated by Kim, Elghobashi \& Sirignano (1993) for solid spheres and by Legendre, Magnaudet \& Mougin (2003) for spherical bubbles, the balance of attractive irrotational effects and repulsive vortical effects leads to the existence of a stable separation distance of the bodies which depends on the Reynolds number. Here we focus our attention on the wake-induced oscillatory motions of nonspherical solid axisymmetric bodies freely falling in a tube. The production of vorticity at the body surface is strongly related to the shape of the body, characterized by its aspect ratio $\chi$. In the unbounded liquid case, the aspect ratio influences significantly the relative weight of the loads acting along the axial and transversal directions of the body, resulting in different types of path (Ern et al. 2007). In the confined situation, it can thus be expected that the aspect ratio will also play an important role in the hydrodynamical interaction with the wall.

The few studies concerned with the effect of confinement on the wake-induced oscillatory motion of a body mainly focused on the identification of the type of path and the corresponding mean horizontal position of the body in the tube. Feng, Hu \& Joseph (1994) investigated numerically the sedimentation of particles of spherical and elliptical cross-section in two dimensions. For Reynolds numbers $\boldsymbol{R e}_{c}$ lower than 60 for $S=0.25$, they observed that the spherical particle displays a rectilinear or weakly oscillatory motion with a mean position corresponding to the channel centreline. Above this value of $R e_{c}$, the body oscillates, first harmonically and then irregularly as $R e$ increases $(R e>300)$. The mean horizontal position of the spherical particle is then off-centre and related to a uniform mean rotation, whereas bodies of ellipsoidal shape assume a centred mean position for all $R e$, provided they do not tumble. The same scenario was observed for other values of $S$, the value of $R e_{c}$ decreasing when the confinement ratio increases. Yu, Phan-Thien \& Tanner (2004) simulated the rectilinear and periodic paths of a sphere falling in a tube with a confinement ratio of $S=0.2$ and a density ratio of 1.5 . They reported three different regimes when the Reynolds number varies: the sphere migrates toward the tube centreline $(R e=20$ and 100); it 
oscillates nearly in a plane about this same axis $(R e=200)$; the helicity of the path increases and the sphere spirals down closer to the tube wall than to the tube axis $(R e=300$ and 400). The simulations by Deloze (2011) on a longer time period also reported, in the same range of parameters, the migration towards the tube axis and the helical character of the periodic path. Moreover, they observed that, for $\operatorname{Re} \approx 350$, vortex shedding occurs at a frequency higher than that of the oscillatory path, leading to a more irregular motion of the sphere. On the experimental side, Figueroa-Espinoza, Zenit \& Legendre (2008) investigated the free rise of an uncontaminated bubble between two parallel plates. They observed that the threshold for path instability decreases with the confinement ratio and related the destabilizing effect of the walls on the bubble path to the attractive potential effect that draws the bubble aside from the centre plane, initiating its periodic oscillation. A quantitative investigation of the impact of confinement on the characteristics of the motion of freely moving solid bodies is thus lacking at present. The aim of this paper is to provide a contribution in this direction.

Since wake dynamics plays a leading role in the kinematics of a freely moving body, it is meaningful to also consider the related situation of the impact of confinement on the wake instability about a fixed solid body. In this case, the presence of confinement was shown to have a stabilizing effect. Below the threshold of wake instability, Tavener (1994) and Maheshwari, Chhabra \& Biswas (2006) have shown that the length of the recirculating wake of a fixed sphere decreases when the confinement ratio is increased. Numerical investigations for fixed two-dimensional cylinders (Chen, Pritchard \& Tavener 1995; Sahin \& Owens 2004) and fixed spheres (Tavener 1994; Cliffe, Spence \& Tavener 2000) placed in an incoming confined flow indicate that for $S<0.5$, wake instability is delayed. For $0.5<S<0.7$, the threshold for wake instability decreases but remains larger than its value in the unconfined case. The restabilizing effect of the walls is related to the vorticity of the boundary layer generated at the wall, which strains the vorticity produced on the body surface. An increase of the Strouhal number characterizing the wake unsteadiness is observed when the confinement is increased. Wake instability is also modified when the fixed body is off-centre in the channel or tube. Zovatto \& Pedrizzetti (2001) and Bhattacharyya \& Maiti (2006) investigated numerically the efforts and the onset of wake instability for cylinders of circular and square cross-sections placed at different transverse positions in a channel with a confinement ratio of $S=0.2$. They observed that wake instability is delayed the more the body approximates the wall. The interaction of the vorticity of the incoming Poiseuille flow and the vorticity generated at the body surface may also result in the inversion of the von Kármán vortex street in the far wake of the body, the vortex generated on one side of the body shifting to the other side (Camarri \& Giannetti 2007, 2010). The structure and the stability of the wake past a fixed body centred in an incoming uniform confined flow will thus also be considered in this paper to shed light on the behaviour of freely moving bodies.

The focus of this paper is on the effect of confinement on the kinematics of freelymoving bodies. The paper is organized as follows. Section 2 describes the experimental and numerical tools used for the investigation. Section 3 traces out the different paths observed experimentally (rectilinear, periodic, erratic) as a function of the Reynolds number and the confinement ratio, for three aspect ratios; the transition from the rectilinear to the periodic motion is also compared to the bifurcations in the wake past fixed bodies placed in an incoming uniform confined flow, which are determined numerically. The effect of the confinement ratio on the mean vertical velocity and on 
the characteristics of the oscillatory motion of the body are analysed in $\S \S 4$ and 5, respectively. The papers ends with a summary and discussion of the results in $\S 6$.

\section{The experimental and numerical tools}

The bodies are released in cylindrical Plexiglas tubes immersed in a glass tank $(1.70 \mathrm{~m}$ high with a square cross-section of $0.4 \mathrm{~m}$ width) containing salted water of density $\rho_{f} \simeq 1010 \mathrm{~kg} \mathrm{~m}^{-3}$ and kinematic viscosity $\nu \simeq 1.020 \mathrm{~mm}^{2} \mathrm{~s}^{-1}$. The tubes have a circular cross-section of diameter $D$ which varies between 9 and $114 \pm 0.1 \mathrm{~mm}$ for a length exceeding $1.1 \mathrm{~m}$ in all cases. They are held vertically (the inclination angle of the tube relative to the vertical is determined with a maximal error of $2 / 1000$ ). The bodies are short-length cylinders of density $\rho_{s} \simeq 1020 \mathrm{~kg} \mathrm{~m}^{-3}$. Their diameters $d$ (respectively heights $h$ ) range from 5 to $20 \mathrm{~mm}$ (respectively $1-5 \mathrm{~mm}$ ) and are measured with an accuracy $\pm 0.01 \mathrm{~mm}$. The body is released with no initial velocity and with its symmetry axis making a small angle to the vertical (less than $10^{\circ}$ ). We verified the release angle had no impact on the characteristics of the paths.

The problem is governed by four dimensionless parameters: the density ratio between the bodies and the fluid, which is here chosen close to one; the aspect ratio $\chi=d / h$, taken to the values 3,6 and 10 , the confinement ratio $S=d / D$ (ratio between the body diameter $d$ and the tube diameter $D)$, determined with an accuracy of $\pm 1 \%$; and the Archimedes number $A r$ defined by $A r=\left(\left(\Delta \rho / \rho_{f}\right) g r_{e q}\right)^{1 / 2} r_{e q} / \nu$, where $r_{e q}$ is the radius of the sphere having a volume equal to that of the body, $\Delta \rho=\left|\rho_{f}-\rho_{s}\right|$ and $g$ is the gravitational acceleration. Note that $A r$ corresponds to a Reynolds number based on a gravitational velocity. Once the mean vertical velocity of the bodies, $U_{m}$, is determined, it can be used to build the Reynolds number, $R e=U_{m} d / \nu$. When the bodies are released in the glass tank corresponding to $S \simeq 1 / 40$ (considered as the unconfined case $S=0$ ), their mean fall velocity corresponds to a Reynolds number $\operatorname{Re}(S=0)=\operatorname{Re}_{0}$ ranging between 100 and 300 (Archimedes numbers $A r$ between 40 and 140), for which both rectilinear and periodic motions are observed (Fernandes et al. 2007). Moreover, it turns out that there is a linear relation between $A r$ and $R e_{0}$ (Fernandes et al. 2007). Since comparison to the case of fixed bodies can only be made via a Reynolds number, we will use in the following preferentially $R e_{0}$ instead of $A r$.

The motion of the bodies was followed by means of two perpendicular travelling cameras. For each tube, a specific calibration was performed to ensure that no optical distortion of the body image due to the curvature of the tube occurred outside a region of $1.5 \mathrm{~mm}$ close to the walls. The image- and signal-processing techniques used to determine the time evolution of the coordinates of the body centre and the angles defining the inclination of its axis are described in detail in Fernandes et al. (2007). The use in this case of two cameras of spatial resolution $2048 \times 2048$ pixels provides an accuracy of $\pm 0.06 \mathrm{~mm}$ for the position and of $\pm 0.75^{\circ}$ for the inclination.

Quantitative information concerning the liquid flow about a body moving along a rectilinear path was obtained by numerical simulations. For various confinement ratios, we performed direct numerical simulations of the flow about a body falling at constant velocity in rectilinear motion. For the sake of simplicity, the body is centred in the tube and a frame of reference fixed with the body is selected. Consequently, the boundary conditions of the problem are a uniform incoming flow and walls moving at the same speed as the incoming flow. Two values of the aspect ratio are considered, $\chi=3$ and 10 , and the following values of the confinement ratio are investigated: $S=0.0494,0.197,0.240,0.331,0.399,0.505$ and 0.666 . We used the finite-volume 
code JADIM; see Legendre \& Magnaudet (1998) for details and validation. For both aspect ratios, the size of the computational domain along the axes of symmetry of the body and the tube was $10 d$ upstream and $15 d$ downstream of the body. The threedimensional grid was obtained by rotating a two-dimensional Cartesian grid about the body and tube axes. The grid consists of 32 elementary volumes in the azimuthal direction, 140 (for $\chi=10$ ) and 162 (for $\chi=3$ ) in the axial direction and a number varying between 50 and 130 depending on $S$ in the radial direction. The grid is refined close to the body and the walls to allow a satisfactory description of the boundary layers for the range of Reynolds numbers investigated $(100 \leqslant R e \leqslant 250)$. The spacing of the grid nodes adjacent to the disk surface is equal to $d / 100$ in both axial and radial directions. The computations are initialized with a uniform axial velocity imposed at the top of the domain, which is also kept constant on the walls. The stability of the flow was investigated by applying a sinusoidal transverse force in a grid cell located in the near wake. This disturbance was imposed for 80 time steps after the initial transient stage and its amplitude was less than $1 \%$ of the final drag force. The results obtained for $S=1 / 20$ and $\chi=3$ for the thresholds of wake instability (see next section) are $158<R e_{c 1}<160$ and $177.5<R e_{c 2}<180$ in agreement with the values of 159.4 and 179.8 from Auguste, Fabre \& Magnaudet (2010). To ensure that numerical results were grid-independent, two-dimensional numerical simulations were performed on a refined grid consisting of 180 (for $\chi=10$ ) and 220 (for $\chi=3$ ) elementary volumes in the axial direction and by multiplying by 1.75 the number of volumes in the radial direction, for $S=0.0494,0.505$ and 0.666 and for $R e=100$ (stationary axisymmetric wake). The differences in drag coefficient obtained with the two grids were in all cases less than $0.8 \%$. Moreover, in the extreme case of a thin body in strong confinement $(\chi=10, S=0.666)$, the values of $R e_{c 1}$ (see next section) calculated with the two grids presented a maximum difference of $4 \%$.

\section{Nature of the path of a body falling in a tube}

The type of path observed experimentally is mapped in the plane of parameters $R e$ and $S$ in figure $1(a-c)$ for the aspect ratios $\chi=3,6$ and 10 , respectively. Rectilinear (periodic) motions are marked out with squares (circles). For each aspect ratio, provided the Reynolds number is lower than a critical value $\operatorname{Re}_{c}(\chi, S)$, the body follows a rectilinear path with its symmetry axis aligned with the vertical direction. For $R e>R_{c}(\chi, S)$, the body displays an oscillatory motion. For $\chi=3$ and $\chi=6$, the threshold for path instability is only weakly modified by the confinement. The transition from the rectilinear to the periodic motion occurs for all $S$ for nearly the same critical Reynolds numbers as for the unconfined case, $\operatorname{Re}_{c}(\chi=3, S=0) \simeq 150$ and $\operatorname{Re}_{c}(\chi=6, S=0) \simeq 130$. In contrast, for thin bodies $(\chi=10) \operatorname{Re}_{c}$ is unaffected by $S$ until $S=0.2(D=5 d)$, but as the confinement ratio is further increased, $R e_{c}$ decreases from $\operatorname{Re}_{c}(\chi=10, S<0.2)=200 \pm 10$ to $\operatorname{Re}_{c}(\chi=10, S=0.5)=100 \pm 10$.

For all $\chi$, when the confinement ratio exceeds a critical value $S>S_{\max }(\chi, R e)$, the amplitude of the oscillatory motion of the body becomes comparable to the radius of the tube and the body starts to impinge on the wall. These cases are denoted by the crosses in figure 1. A quantitative investigation of these paths is beyond the scope of this work but it is worth pointing out that they appear irregular to the eye, the contact with the wall occurring irregularly. The figures indicate that $S_{\max }$ decreases with the aspect ratio, a trend consistent with the decrease of $\operatorname{Re}_{c}$ with $S$ for thin bodies. For a given Archimedes number $A r$, a body displaying an oscillatory motion in an 


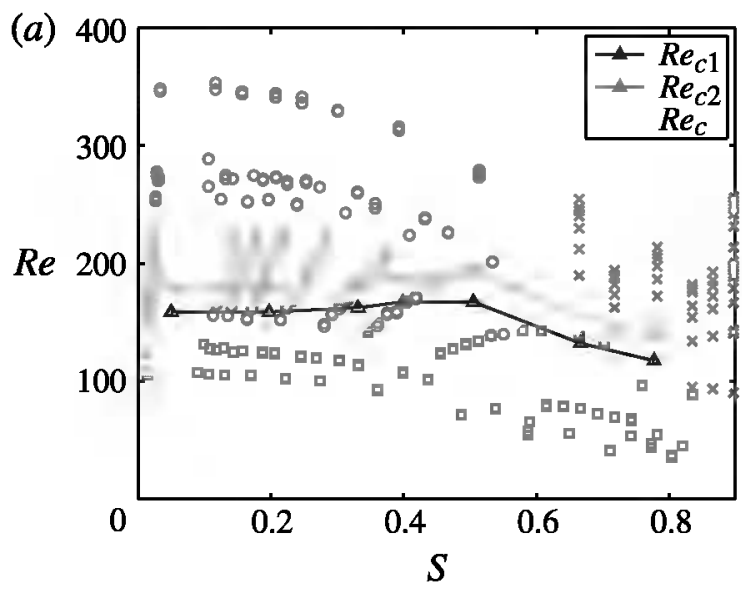

(b)
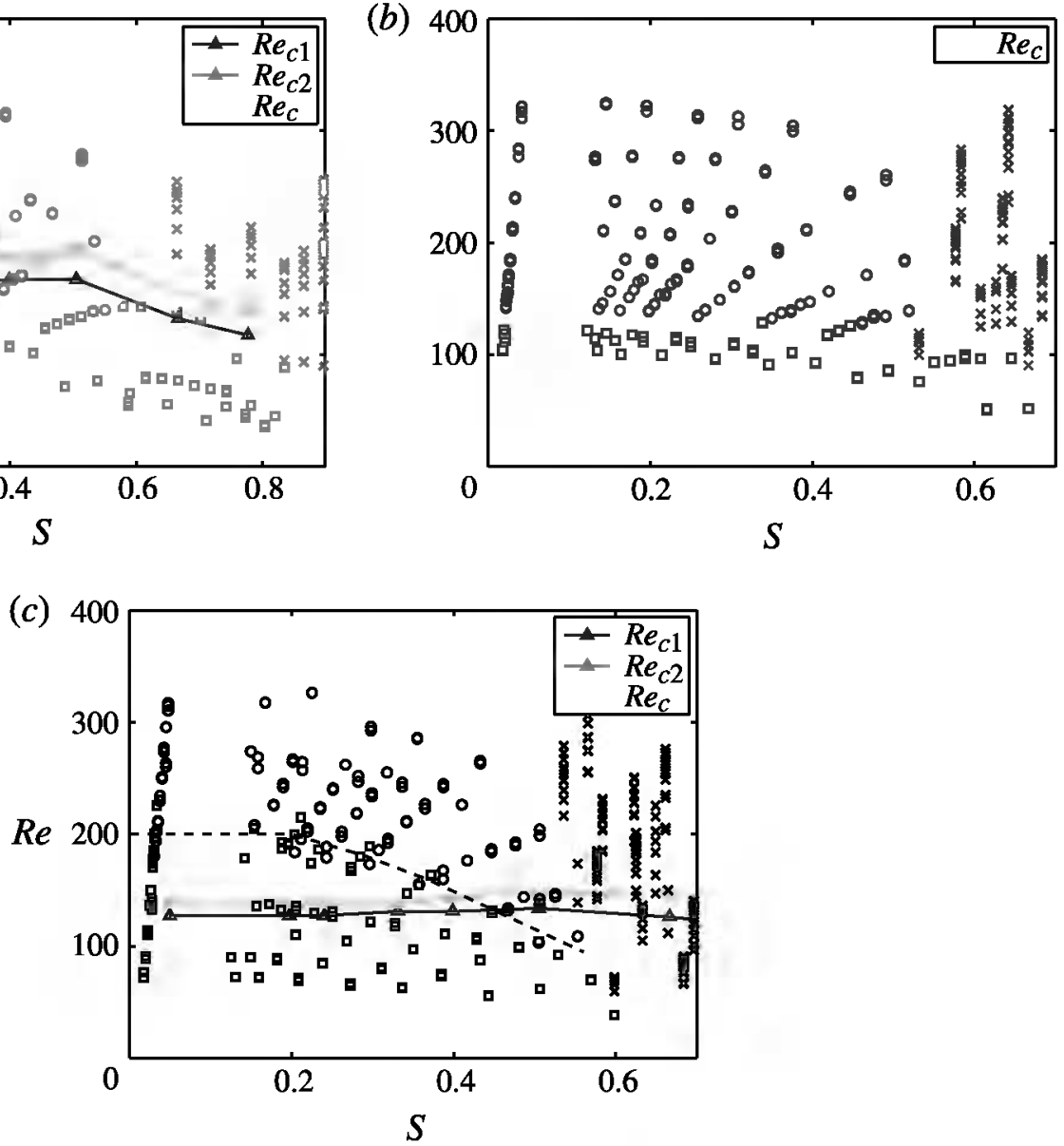

FIGURE 1. Nature of the path displayed by bodies with aspect ratio $\chi$ as a function of $\operatorname{Re}$ and $S . \square$, rectilinear paths; o, periodic paths; $\times$, cases where the body touches the tube from time to time. The black and grey curves correspond to the evolutions of the critical Reynolds numbers $\boldsymbol{R e}_{c 1}$ and $R e_{c 2}$ for the wake instability of the fixed body when the confinement ratio varies, obtained by numerical simulations: $(a) \chi=3$; $(b) \chi=6$ (no numerical result in this case); (c) $\chi=10$.

unconfined medium still displays a periodic motion in the presence of confinement for $\chi=10$, while a body with $\chi=3$ regains a rectilinear motion for sufficiently large $S$.

The onset of path instability for a body falling rectilinearly in a tube can be compared with the thresholds of wake instability past the same body held fixed in a uniform flow imposed by the moving walls of the tube. As mentioned in $\S 2$, this situation was investigated by numerical simulations for various confinement ratios. We will restrict our attention to the aspect ratios $\chi=3$ and $\chi=10$. In an unconfined medium, the wake instability of bodies with such aspect ratios was investigated in detail by Auguste (2010) and Auguste et al. (2010). They identified two different sequences of bifurcations in a short range of Reynolds numbers depending on the body aspect ratio. We focus here on the first bifurcation at $R e_{c 1}$, corresponding to the loss of axial symmetry of the wake, and on the second bifurcation at $\operatorname{Re}_{c 2}$, beyond which the wake becomes unsteady. The results are shown with solid lines in figures $1(a, c)$ and 2. For $\chi=3$, the thresholds $\operatorname{Re}_{c 1}$ and $\boldsymbol{R e}_{c 2}$ are delayed when the confinement ratio is increased until $0.4 \leqslant S \leqslant 0.5$, beyond which they start to decrease; $R e_{c 1}$ (respectively $R_{c 2}$ ) decreases from 167.5 (respectively 192.5) \pm 2.5 to 132.5 (respectively 157.5) \pm 2.5 at $S=0.666$. For $\chi=10$, the changes of $R e_{c 1}$ and $R e_{c 2}$ with $S$ follow the 


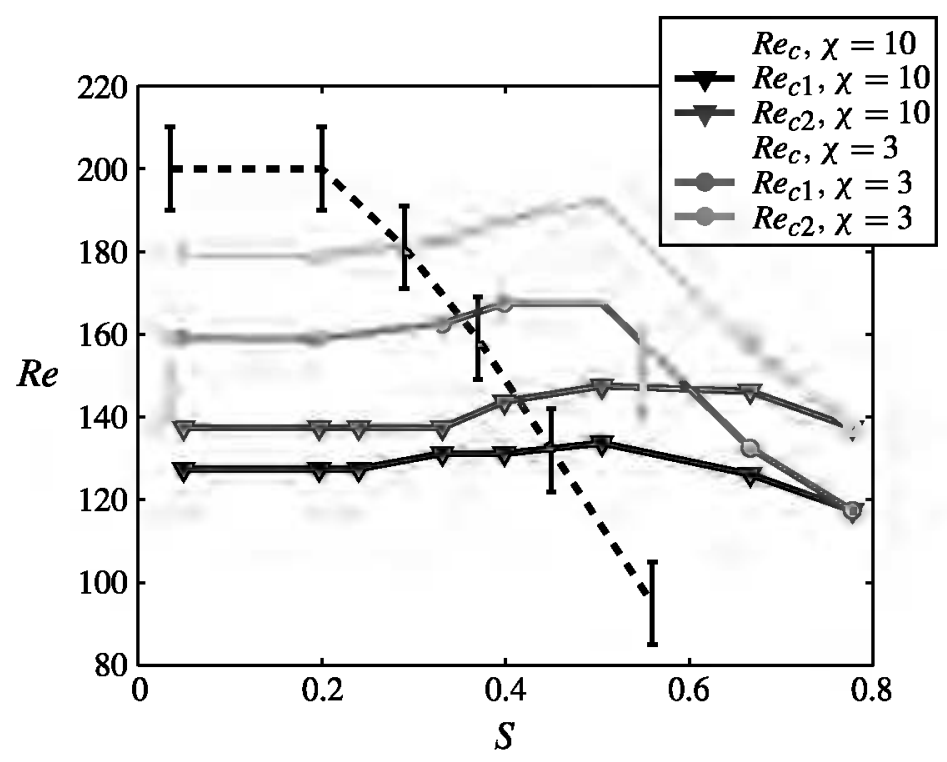

FIGURE 2. Critical Reynolds numbers as a function of the confinement ratio $S$ for $\chi=3$ and $\chi=10$. Solid lines, $R e_{c 1}$ corresponding to the loss of axial symmetry and $R e_{c 2}$ to the loss of stationarity of the wake about a body moving at constant velocity in a tube (numerical simulations); dashed lines, $\boldsymbol{R e}_{c}$ corresponding to the onset of a periodic motion for the freely moving body (experiments).

same trend but are weaker, increasing until $R e_{c 1} \simeq 134\left(\operatorname{Re}_{c 2} \simeq 148\right)$ at $S=0.5$ and then decreasing to $\sim 126$ (146) at $S=0.666$. Tavener (1994) and Cliffe et al. (2000) observed the same trend in the case of a sphere embedded in a Poiseuille flow: $\operatorname{Re}_{c 1}$ first increases and then decreases when the confinement ratio increases. Zovatto \& Pedrizzetti (2001) related this effect to the interaction of the vorticity produced at the surface of the body with the vorticity produced at the wall, which have opposite signs. Figure 2 also shows the evolution with $S$ of $\operatorname{Re}_{c}$ for the path instability of bodies with aspect ratios $\chi=3$ and 10 . For thick bodies, path instability slightly anticipates the critical Reynolds number for the break-up of axial symmetry in the wake of the body held fixed, $\operatorname{Re}_{c}(\chi=3, S) \simeq 150$ for $S \leqslant 0.57$, which is $\sim 10 \%$ lower than $\operatorname{Re}_{c 1}$. In contrast, for a body with $\chi=10$, the appearance of significant oscillations of the body is notably delayed provided the confinement is sufficiently weak: for $S \leqslant 0.2$, $\operatorname{Re}_{c}(\chi=10, S)$ is $\sim 60 \%$ larger than $\operatorname{Re}_{c 1}$ but then decreases with $S$ until $\operatorname{Re}_{c} \simeq R e_{c 1}$ for $S \simeq 0.45$. In the unconfined case $(S=0)$, the numerical simulations of Auguste (2010) revealed that various non-rectilinear paths presenting irregular oscillations of low amplitude exist in the range $\left[R e_{c 1}, \operatorname{Re}_{c}\right]$ for $\chi=10$. It is reasonable to expect that this regime of low-amplitude oscillations, which are difficult to detect experimentally (for a discussion about this regime of oscillations, see Ern et al. 2012), exists in the presence of weak confinement, but in any case its extension clearly decreases when the confinement ratio increases. When the confinement is strong enough, $S \geqslant 0.45$ for $\chi=10$, path instability occurs at a Reynolds number lower than that of the wake instability of the fixed body. However, for these confinement ratios, the body starts to impinge on the walls at slightly higher $R e$. Note also that for a body with $\operatorname{Re}_{0}$ in the range $\left[\operatorname{Re}_{c 1}(\chi=10, S=0), \operatorname{Re}_{c}(\chi=10, S=0)\right]$, increasing the confinement leads to a destabilization of its rectilinear motion, since a regular periodic motion of significant amplitude sets in above a critical value of $S$.

Figure 3(a) shows a three-dimensional view of the motion of a disk falling in a tube. The projections of the path in two perpendicular vertical planes are also 
(a)

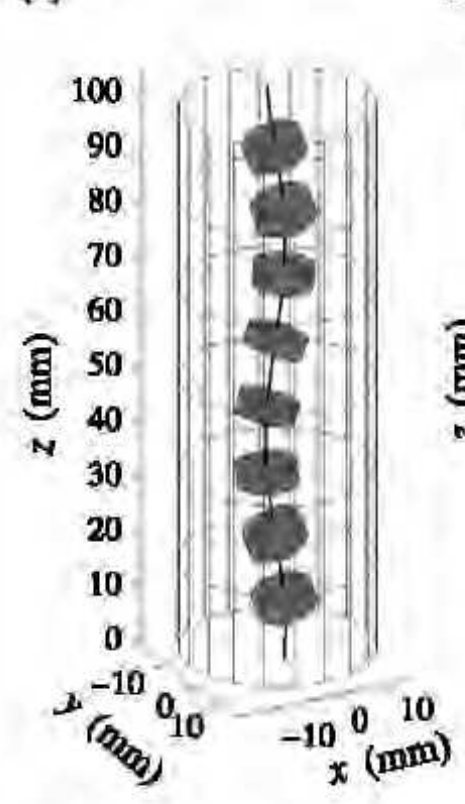

(b)

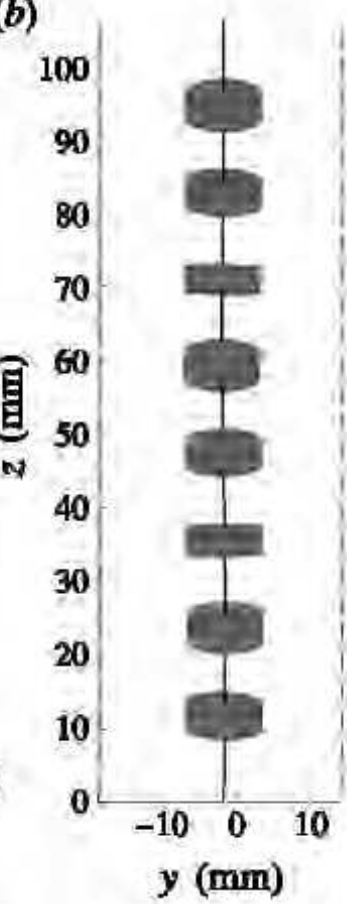

(c)

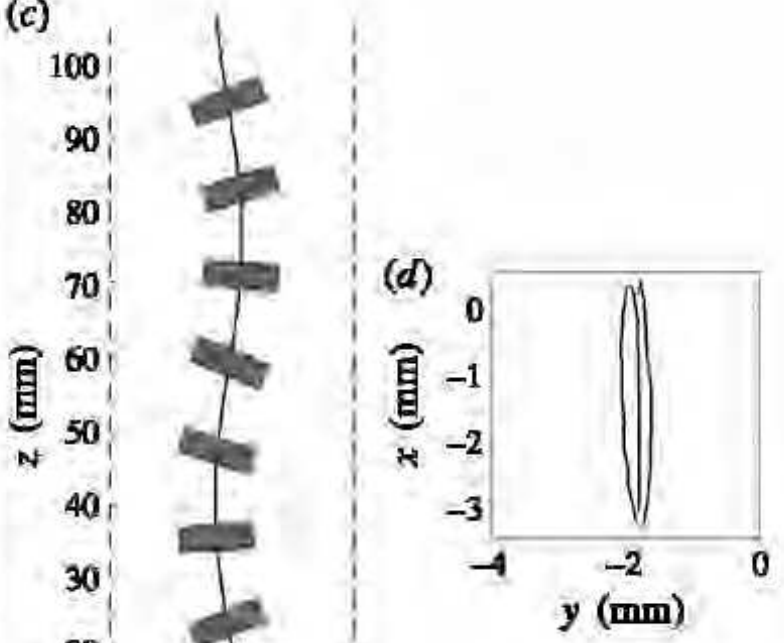

FIGURE 3. Ilustration of roughly one period of the motion of a disk falling in a tube. (a) Three-dimensional view: the grid represents the wall of the tube. $(b, c)$ Two perpendicular views: -, path of the disk; - , wall of the tube. (d) Top view of the path $(\chi=3, S=0.318$, $\left.\operatorname{Re}(S=0.318)=243, \operatorname{Re}_{0}=256, d=10,5 \mathrm{~mm}, D=33 \mathrm{~mm}\right)$.

presented (figure $3 b, c)$ : in $(c)$, in the plane hereafter called the principal plane of oscillation, the position of the body, as well as its inclination relative to the vertical, displays periodic oscillations; in (b), the trajectory is almost rectilinear. No preferential orientation of the plane of principal oscillations was observed in the laboratory frame. In an unconfined liquid, the periodic paths measured experimentally by Fernandes et al. (2007) in the range of parameters investigated here are nearly two-dimensional; in the horizontal plane, the path presents an ellipsoidal trace of low eccentricity in most of the cases (as also observed in figure $3 d$ ). Letting $\tilde{x}$ be the amplitude of the motion along the principal direction of the oscillations and $\tilde{y}$ its amplitude along a perpendicular direction, the eccentricity of the path is defined by $e=\tilde{y} / \tilde{x}$. In an equivalent manner, the eccentricity could have been defined using the projections of the inclination angle of the body in the two planes. Identical values are obtained with both definitions. The numerical simulations of Auguste (2010) provided instead purely two-dimensional paths of amplitudes very close to those measured experimentally along the principal direction of oscillation. The eccentricities of the paths measured in the presence of confinement ane presented in figure 4 when $R e$ and $S$ are varied. The results are in line with those of Fernandes et al. (2007) in the absence of confinement (shown with a dashed line for each aspect ratio). A majority of trajectories present low eccentricity $(e \leqslant 0.25)$ and can be assimilated to a plane zigzag motion. This is in particular the case for all the periodic paths of thin bodies $(\chi=10)$. For $\chi=3$ and $\chi=6$, larger eccentricity is observed for lower Reynolds numbers, for which the amplitude of the oscillations is weaker. A sharp decrease in eccentricity occurs when the Reynolds number is increased. For $R e \geqslant 250$ and strong confinement $(S \approx 0,5)$, we observed that some paths for $\chi=3$ and $\chi=6$ display a helical shape $(0,3 \leqslant e \leqslant 1)$. In the following, we will restrict our attention to the analysis of the effect of $R e$ and $S$ on 


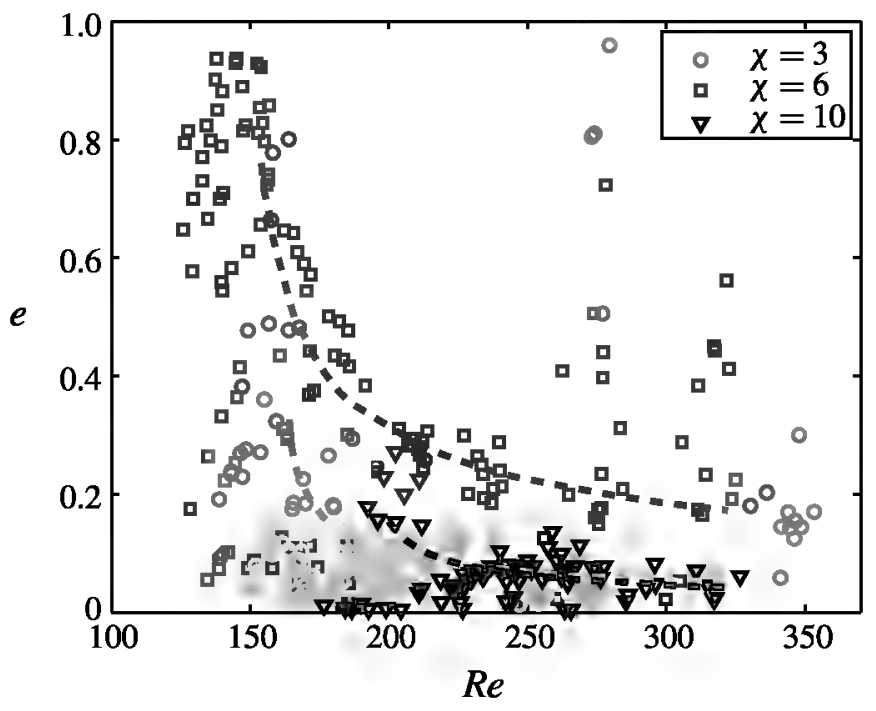

FIGURE 4. Eccentricity of the observed periodic paths as a function of the Reynolds number $R e$, for various confinement ratios $S$. The dashed lines correspond to the mean values of the eccentricity measured in the unbounded case (Fernandes et al. 2007).

the characteristics of the planar zigzag motions (i.e. for the periodic paths presenting a low eccentricity, $e \leqslant 0.25)$.

\section{Mean radial position and mean vertical velocity of the body}

\subsection{Mean radial position of a body falling in a tube}

The bodies were released at various radial positions in the tube in order to investigate the radial migration of the bodies during their fall and the possibility of observing off-centre motions. For a given body released in a given tube, different initial radial positions were chosen randomly between 0 and $D / 4$ from one experiment to the other. After a short transient following the release of the body from rest, the body kept a mean radial position in the tube. A slight horizontal drift about this mean radial position could be observed, but in all cases it had no preferential direction and was lower than $3 \%$, which is comparable to the drift occurring in the unconfined case (Fernandes et al. 2007). We define $\Delta_{w}$ to be the horizontal distance between the centre of gravity of the body and the wall, averaged out over the path, transient excluded. Figure 5 presents the values of $\Delta_{w} / d$ for all the paths recorded when the confinement ratio $S$ was varied. The body is centred when $\Delta_{w} / d=(2 S)^{-1}$. When $\Delta_{w}=d / 2$, the body touches the tube. For low confinement ratios, the bodies do not select any preferential position in the tube, but they keep a position sufficiently far from the wall: for $S<0.4, \Delta_{w} / d>1$, indicating that there is in all cases at least half a diameter between the border of the body and the wall. Provided the body is sufficiently far from the wall, the body may keep its radial position and no significant radial drift is observed. For confinement ratios larger than $S=0.4$, figure 5 shows that the mean positions of the bodies are nearly centred in the tube (differences lower than $3 \%$ ), for both rectilinear and periodic motions and for any body aspect ratio.

\subsection{Modification of the mean fall velocity due to the confinement}

The effect of the confinement ratio $S$ on the body motion can be investigated at fixed $A r, \chi$ and $\rho_{s} / \rho_{f}$ by releasing the same body in tubes of decreasing diameters. We observe that the mean vertical velocity of the body, $U_{m}(S)$, decreases with $S$, as 


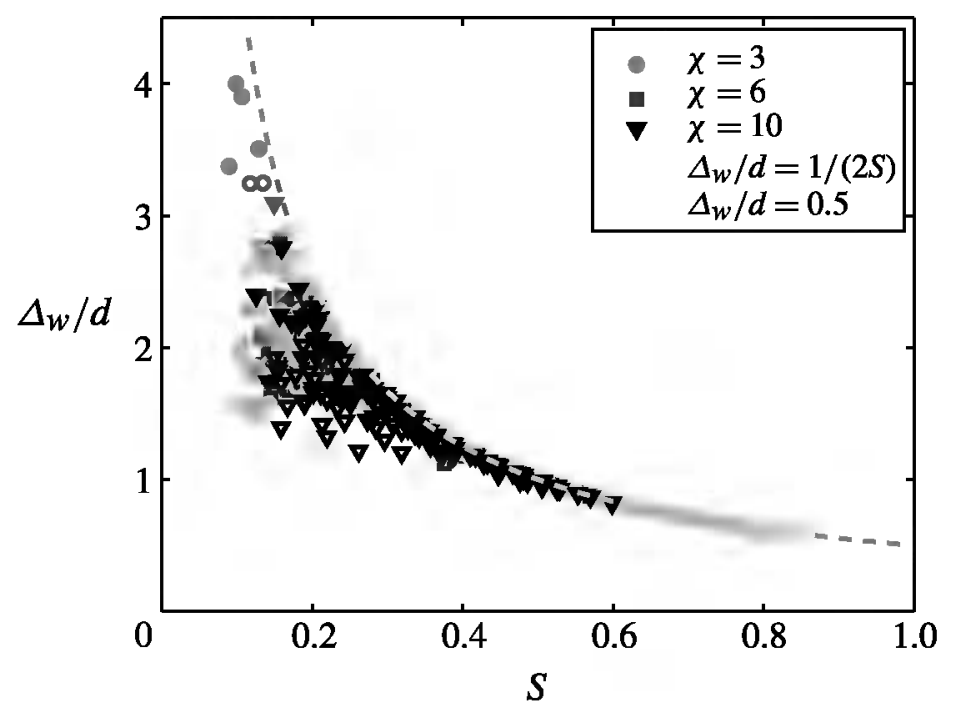

FIGURE 5. Mean distance between the centre of gravity of the body and the wall, $\Delta_{w}$, normalized with the body diameter $d$, as a function of the confinement ratio. Filled and open symbols correspond to bodies having rectilinear and periodic paths, respectively. The black dashed line indicates the distance at which the body touches the wall, the dashed grey one the distance corresponding to a centred body.
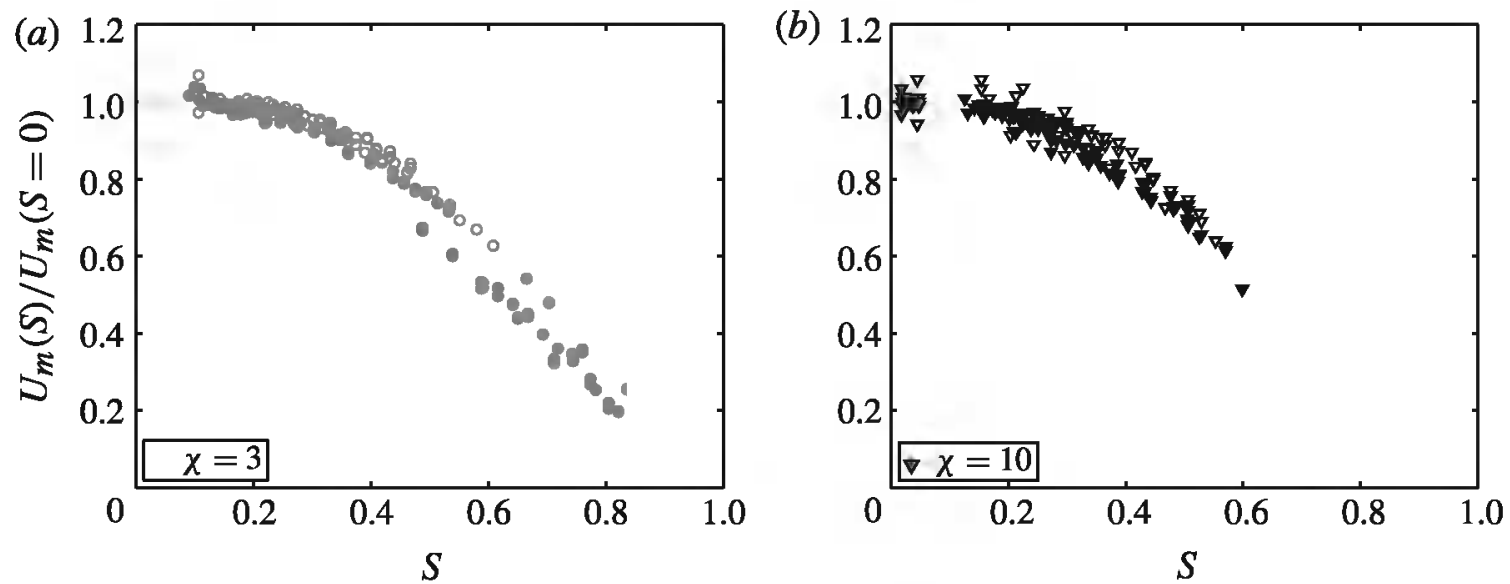

FIGURE 6. Mean vertical velocity, $U_{m}(S)$, normalized with its value in the absence of confinement, $U_{m}(S=0)$, as a function of the confinement ratio $S:(a) \chi=3$; $(b) \chi=10$. Filled (open) symbols correspond to rectilinear (periodic) paths.

illustrated in figure $6(a, b)$ for $\chi=3$ and $\chi=10$, where the mean fall velocity is normalized with its value in the unconfined case, $U_{m}(S=0)$. For all aspect ratios, we observe that the decrease is weaker for the periodic paths than for the rectilinear paths. For confinement ratios lower than 0.2 (i.e. the diameter of the tube being at least 5 times larger than the body diameter), the influence of the confinement is weak, the difference in mean fall velocity being of the order of magnitude of the variability from one experiment to the other. At larger confinement ratios, the decrease in mean fall velocity becomes stronger.

A characteristic feature of the confinement effect is the corresponding increase of the drag coefficient: see for instance Clift, Grace \& Weber (1978), Wham et al. (1996) and Chhabra, Agarwal \& Chaudhary (2003). The drag coefficient is defined 


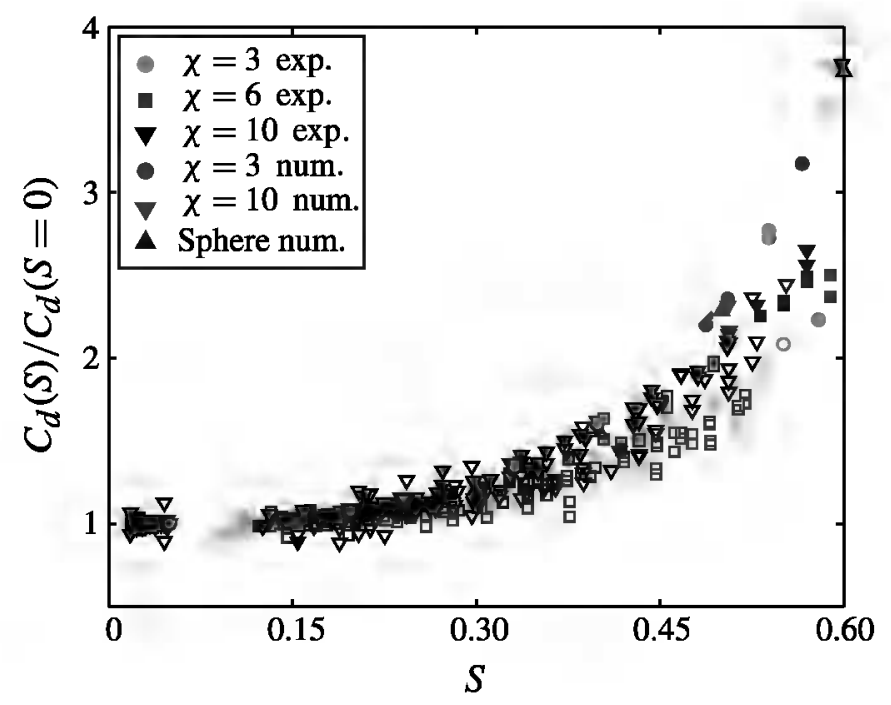

FIGURE 7. Drag coefficient, $C_{d}$, normalized with its value in the absence of confinement, $C_{d}(S=0)$, as a function of the confinement ratio $S$. Filled (open) symbols, rectilinear (periodic) paths. Also presented are the drag coefficients obtained from the numerical simulations with fixed bodies with $\chi=3$ and $\chi=10$ and the results for a sphere at $\operatorname{Re}=100$ from Wham, Basaran \& Byers (1996). $C_{d}$ is based on the mean vertical velocity $U_{m}(S)$.

by $C_{d}(S)=\mathscr{M} g /\left(1 / 2 \rho_{f} \mathscr{A} U_{m}^{2}\right)$, where $\mathscr{M}$ is the mass of the disk, $\mathscr{M}=\rho_{s} \mathscr{A} h$ and $\mathscr{A}=\pi d^{2} / 4$. Figure 7 presents the evolution with $S$ of the drag coefficient $C_{d}$, based on $U_{m}(S)$ and normalized with the drag coefficient of the body in the absence of confinement $C_{d}(S=0)$. For larger confinement ratios a sharp increase in $C_{d}$ occurs, corresponding to the rectilinear paths of thick bodies with $\chi=3$ and to the jump down of their mean fall velocity visible in figure $6(a)$ (for these values of $S$, thin bodies oscillate, touching the walls from time to time). For thick bodies, this regime may be related to an increase of viscous friction on their lateral surface (parallel to the stream and the walls). This trend is in agreement with the numerical simulations for a sphere by Wham et al. (1996), as shown in figure 7 (we normalized their results with the value they obtained for $S=0.15$ ). It is also in agreement with the evolution of the drag coefficient with $S$ (dark circles in figure 7) obtained from our numerical simulations for a fixed body with $\chi=3$ and $R e=100$ (stable wake).

An insight into the decrease of the mean fall velocity of a body when the confinement ratio increases can be gained by investigating the modification of the structure of the body wake with $S$. This was performed by means of numerical simulations for bodies held fixed in an incoming confined uniform flow. The intensity of the flow in the attached region of the axisymmetric stationary wake can be characterized by the length $L_{w}$ of the recirculating region (normalized with the body diameter) and by the maximal velocity $V_{w}$ (normalized with the velocity $U_{m}(S)$ ) taken on the axis of symmetry of the body (see figure 14 of Fernandes et al. 2007). For bodies in rectilinear motion in an unconfined medium, Fernandes et al. (2007) showed that these quantities increase with the aspect ratio of the body, and that for $R e=R e_{c 1}$ and $S=0$,

$$
\left(\frac{V_{w}}{U_{m}}\right)=0.62\left(1+\frac{1}{\chi}\right)^{-1} .
$$


(a)

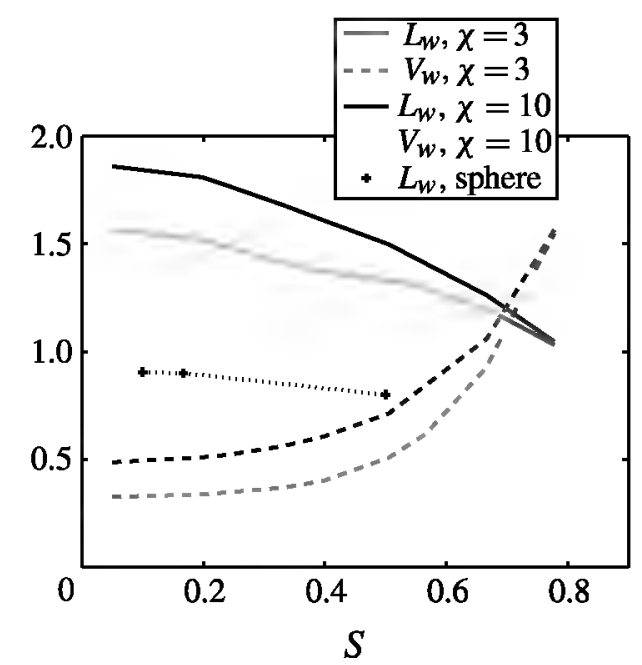

(b)

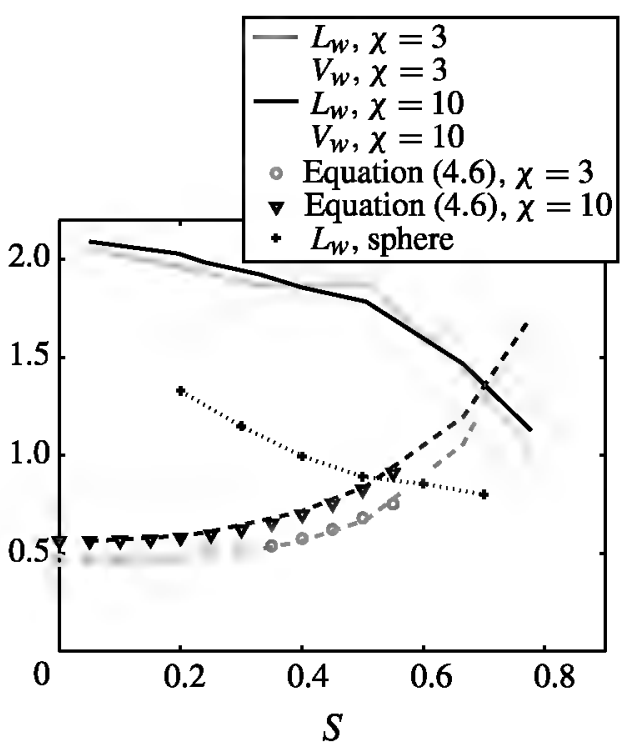

FIGURE 8. Effect of the confinement ratio $S$ on the dimensionless quantities, $L_{w}$ and $V_{w}$, characterizing the attached wake past a body (with $\chi=3$ and 10) held fixed in an incoming confined uniform flow (numerical simulations): $(a) R e=100$, case of the sphere from Maheshwari et al. (2006), (b) $R e=R e_{c 1}$, case of the sphere from Tavener (1994).

Moreover, they introduced the Reynolds number

$$
\operatorname{Re}^{*}(S=0)=\operatorname{Re} \frac{0.62 \chi}{(1+\chi)}
$$

which for $R e=R e_{c 1}$ can be interpreted as $R e^{*}=V_{w} d / \nu$ and which has the remarkable property that the thresholds of wake instability for a fixed body in an unbounded uniform flow are $\operatorname{Re}_{c 1}^{*}(S=0) \simeq 72$ and $\operatorname{Re}_{c 2}^{*}(S=0) \simeq 78$, for any $\chi$. This result shows that the intensity of the attached wake (characterized by $V_{w}$ ), which increases as the body becomes thinner ( $\chi$ increases) at given $R e$, is a reliable marker of the wake state, anticipating in particular the decrease of the thresholds of wake instability with the aspect ratio. Figure 8 now shows for two Reynolds numbers, $\operatorname{Re}=100$ and $R e=\operatorname{Re}_{c 1}(\chi, S)$, and two aspect ratios, $\chi=3$ and $\chi=10$, the evolutions of $L_{w}$ and $V_{w}$ with the confinement ratio $S$. In all cases, $V_{w}$ increases with $S$, whereas $L_{w}$ decreases. The modification of the attached wake with increasing $S$ is illustrated in figure 9 for a body with $\chi=10$ at $\operatorname{Re}=100$. The evolution of $V_{w}$ with $S$ can be fitted by an empirical function $g(S)$, in order to generalize the relation (4.1) for $R e=R e_{c 1}$ and $S \leqslant 0.6$,

$$
\left(\frac{V_{w}}{U_{m}}\right)=f(\chi) g(S) \quad \text { with } f(\chi)=\frac{0.62 \chi}{(1+\chi)} \text { and } g(S)=1+3.7 S^{3} \text {. }
$$

We now use the function $g(S)$ to extend the definition (4.2) of the Reynolds number $R e^{*}$ for $S \leqslant 0.6$,

$$
\operatorname{Re}^{*}(S)=\operatorname{Re}^{*}(S=0) g(S)=\operatorname{Re} \frac{0.62 \chi}{(1+\chi)}\left(1+3.7 S^{3}\right),
$$

which again in the particular case of $R e=R e_{c 1}$ can be written as $\operatorname{Re}^{*}=V_{w} d / \nu$. For each experiment, the value of the Reynolds number $R e^{*}$ can be calculated. The evolution of the Reynolds number with $S$ is plotted in figure $10(a)$ for $\chi=3$ 

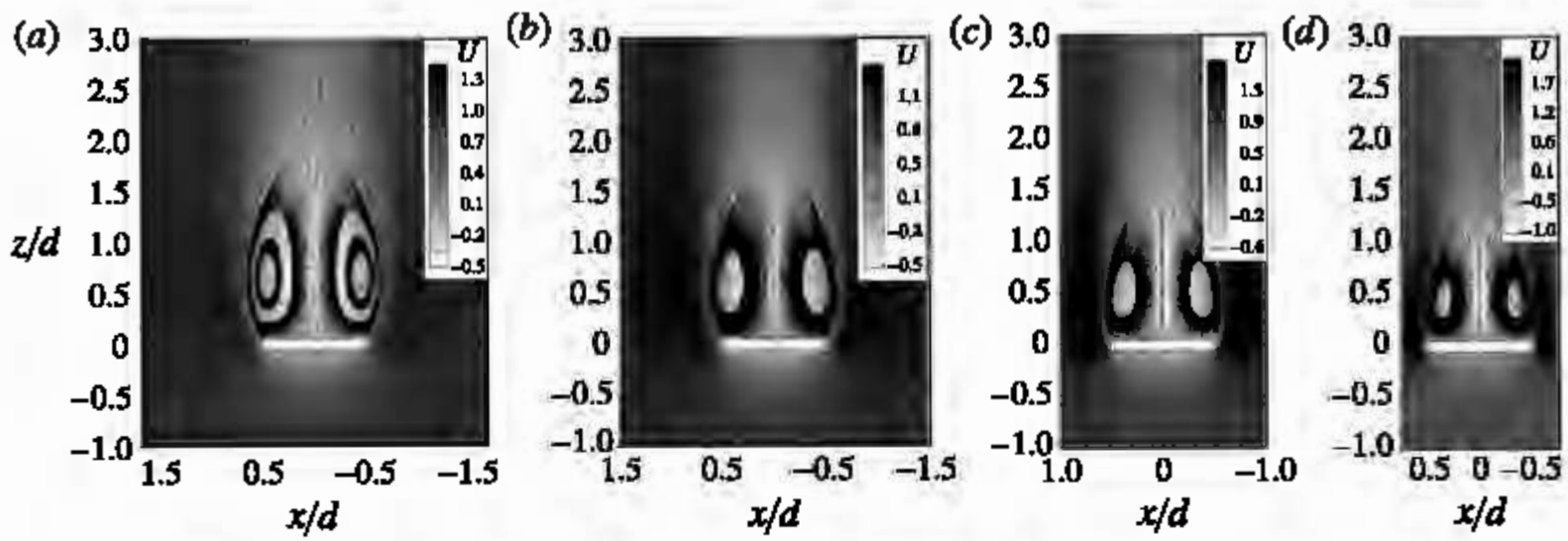

FIGURE 9. Wake of a body with $\chi=10$ at $R e=100$. Streamlines and norm of the axial velocity obtained by numerical simulations for various confinement ratios: (a) $S=1 / 20$; (b) $1 / 3 ;(c) 1 / 2 ;(d) 2 / 3$. Note that (a) displays the results in the vicinity of the body and does not show the whole computational domain.
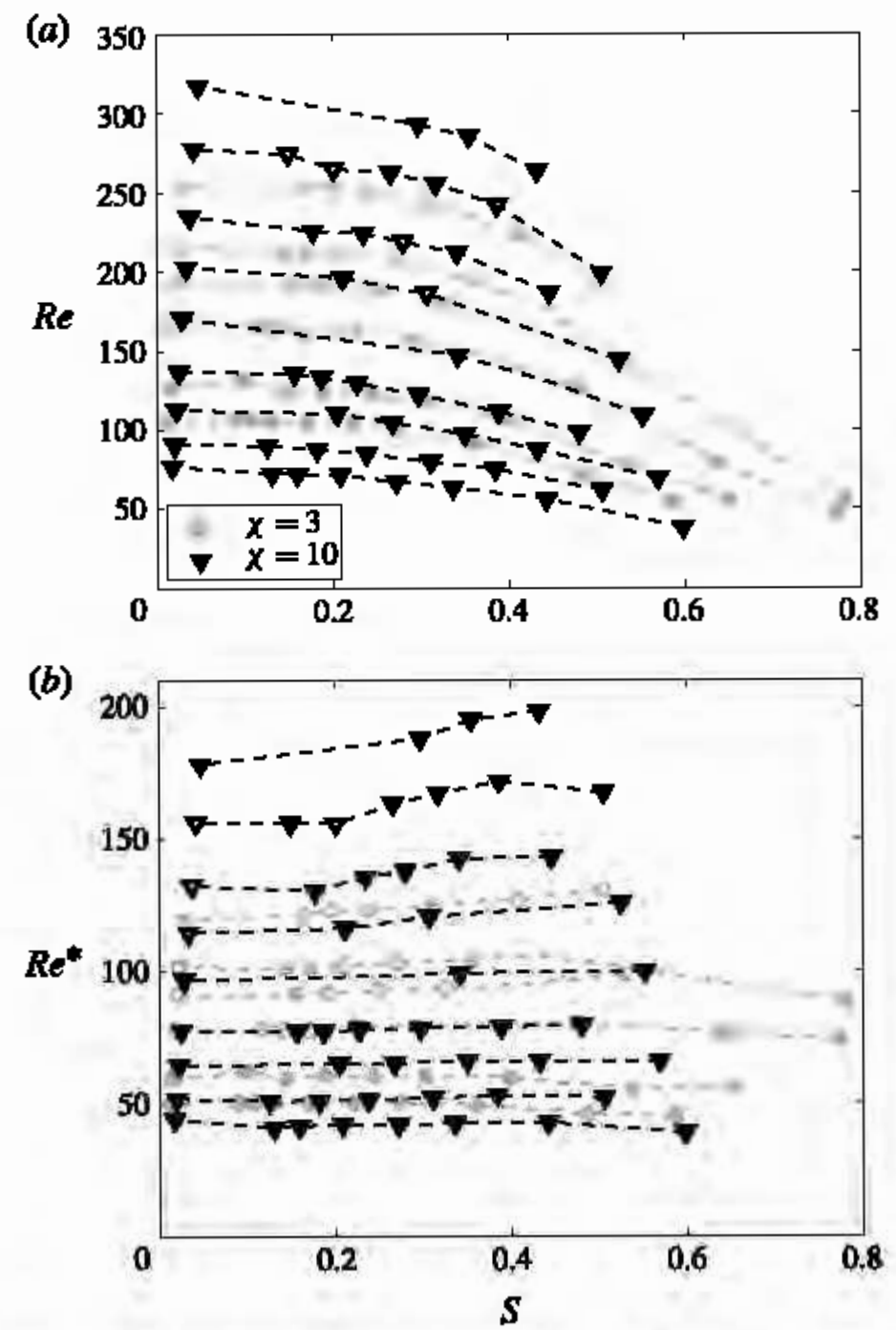

FIGURE 10. Evolution of the Reynolds numbers $R e$ and $R e^{*}$ with the confinement ratio $S$ for bodies with $\chi=3$ and $\chi=10:(a) \operatorname{Re} ;(b) R e^{*}$. Each dashed line corresponds to a body and so to an Archimedes number, whose value can be retrieved from the value of $\operatorname{Re}(S \simeq 0)$ using Fernandes at al. (2007). Filled (open) symbols correspond to rectilinear (periodic) paths. 

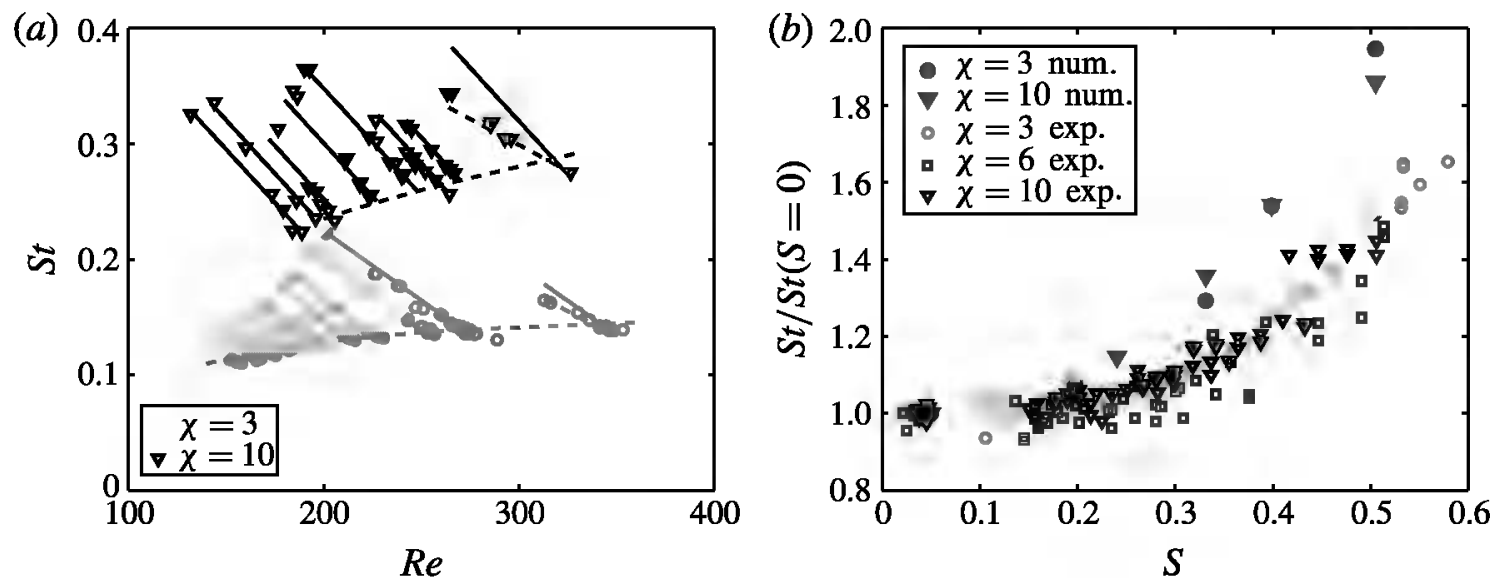

FIGURE 11. Strouhal number $S t$ as a function of $(a)$ the Reynolds number $R e$ and $(b)$ the confinement ratio $S$. (a) Solid lines, (5.1), exhibiting for a given $A r$ the increase of $S t$ when $S$ increases; dashed lines, the mean values of $S t$ measured for $S=0$. $(b) S t$ is normalized with $\operatorname{St}(S=0)$. Also shown for comparison the Strouhal numbers of the wake instability of fixed bodies (numerical simulations).

and $\chi=10$. The decrease of $\operatorname{Re}$ with $S$ for a given body (and thus a given Archimedes number $A r)$ is outlined with a dashed line. Figure $10(b)$ shows the corresponding evolution of $\operatorname{Re}^{*}$ with $S$. The dashed lines again bring together the values for a given Archimedes number, pointing out that for the rectilinear and periodic paths, $R e^{*}$ remains almost constant with $S$ provided the oscillations are not too large. This remarkable result indicates that the change induced by the presence of confinement on the wake structure at the threshold of wake instability for a fixed body, which is accounted for by (4.3), turns out to be a reliable marker of the effect of the confinement on the mean fall velocity of a freely moving body. This result suggests that the mean vertical velocity of the body adjusts to maintain for all the confinement ratios an almost constant intensity in the recirculating wake, characterized as a first approximation by the parameter $R e^{*}$. Note also that, for any confinement ratio, the corresponding value of $R e$ can be obtained from relation (4.4) once $R e$ is known in a single configuration (case $S=0$ included). We now turn our attention to the effect of the confinement ratio on the characteristics of the periodic motion.

\section{Characteristics of the oscillatory motion}

\subsection{Frequency of the periodic path}

We first focus our attention on the frequency $f$ of the oscillations in position and inclination of the body. Figure 11(a) presents the values of the Strouhal number $S t=f d / U_{m}$ as a function of the Reynolds number $R e$, for bodies with aspect ratios $\chi=3$ and 10 and various confinement ratios. Only a few cases are presented to avoid overloading the figure. For each aspect ratio, the quasi-horizontal dashed line shows the behaviour of $S t$ in the absence of confinement $(S=0)$. For a given body (given $\operatorname{Re}_{0}=\operatorname{Re}(S=0)$ and $\left.\chi\right)$, we observe that increasing the confinement ratio $S$ induces a decrease of $R e$ and an increase of St. This trend is exhibited by the solid lines spiking upwards from the dashed lines corresponding to $S=0$, forming a fishbone pattern for each $\chi$. These solid lines correspond to the empirical relation

$$
S t=S t_{0}+\alpha(\chi)\left(R e_{0}-R e\right)
$$



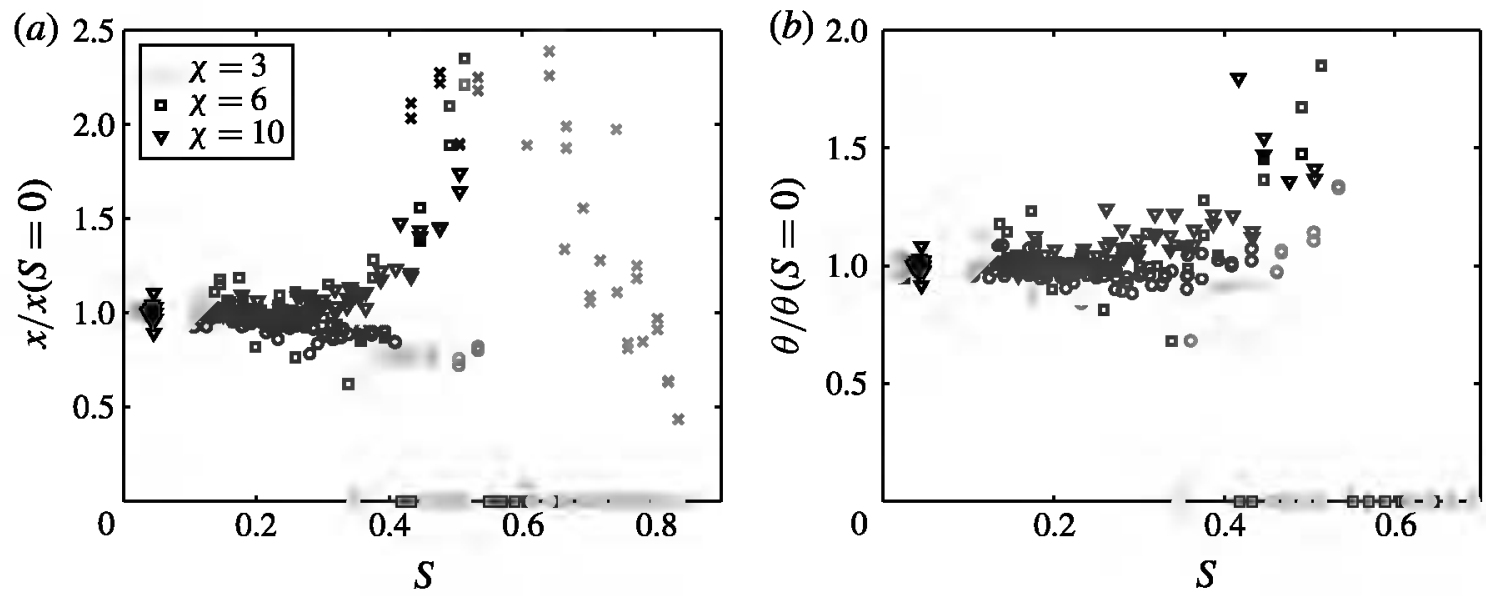

FIGURE 12. Amplitude of the oscillations (normalized with the amplitude in the absence of confinement) as a function of $S$ : (a) horizontal position; $(b)$ inclination $(\chi=3,6$ and 10$)$. The crosses correspond to the cases where the body briefly touches the tube.

where $S t_{0}=S t\left(R_{0}\right)$ is the Strouhal number observed in the absence of confinement and $\alpha=0.0012$ for $\chi=3$ and 0.00185 for $\chi=10$. An interesting feature of this relation is that the confinement ratio does not appear explicitly but its effect on $S t$ is accounted for by the Reynolds number $R e$. Note that for $R e>300$ the prediction obtained with (5.1) is improved by considering the value $\alpha=0.0009$ for both aspect ratios. For all the bodies, figure $11(b)$ presents the evolution of $S t(S)$, normalized with the value $S t(S=0)$ measured in the absence of confinement, as a function of the confinement ratio. This evolution may be compared to the evolution of $S t_{f x x}(S) / S t_{f i x e}(S=0)$, corresponding to the instability of the wake past a fixed body in the presence and absence of confinement, obtained from the numerical simulations at $\operatorname{Re}_{c 2}(\chi, S)$ and $\operatorname{Re}_{c 2}(\chi, S=0$ ) (figure $11 b)$. The confinement leads to a stronger increase of the Strouhal number $S t$ in the case of fixed bodies. For a fixed twodimensional cylinder embedded in a Poiseuille flow, $S t$ was observed to increase even more strongly with $S$, becoming three times larger for $S \approx 0.5$ than in the unconfined case (Chen et al. 1995; Sahin \& Owens 2004).

\subsection{Amplitude of path oscillations}

The evolution with the confinement ratio $S$ of the amplitude of oscillation of the horizontal position of the centre of gravity of the body (normalized with the amplitude in the absence of confinement) is displayed in figure 12(a). For thick bodies with $\chi=3$, the amplitude decreases with $S$ until the bodies restabilize and follow a rectilinear path. Concerning the oscillations of the body inclination, figure 12(b) shows that for $\chi=3$ the inclination amplitude is only weakly modified by the confinement. In contrast, for thin bodies with $\chi=10$, the amplitudes of the horizontal displacement and the inclination increase strongly with $S$ until the body touches the tube; the crosses in figure $12(a)$ indicate when this situation occurs. The aspect ratio $\chi=6$ combines the two behaviours. The type of behaviour depends on the Archimedes number of the body or, equivalently, on the Reynolds number $R e_{0}$ corresponding to its fall in an unbounded liquid. For bodies with $\operatorname{Re}_{c}(\chi=6)<R e_{0}<230 \pm 10$, the confinement induces a stabilization of the periodic motion (the amplitudes of the oscillations in displacement and inclination decrease until $S \simeq 0.4$ ), until the body regains a rectilinear motion, as occurs for thick bodies $(\chi=3)$. For larger $R e_{0}$, however, the amplitude of the oscillations is amplified by the confinement, as observed for thin 

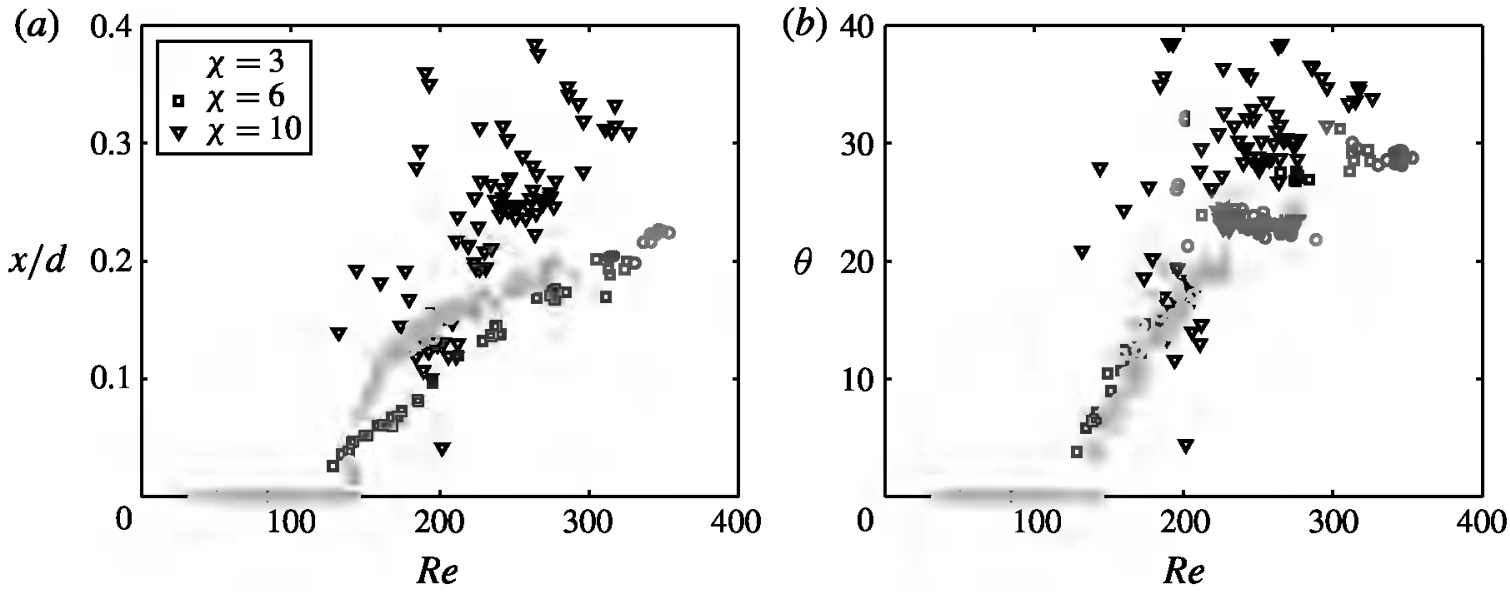

FIGURE 13. Amplitude of the oscillations as a function of the Reynolds number, for various confinement ratios: $(a)$ horizontal position normalized with the body diameter; $(b)$ inclination $(\chi=3,6$ and 10$)$.

bodies $(\chi=10)$. The evolution of the amplitude of oscillations with the Reynolds number $R e$ is displayed in figure 13 for all the confinement ratios investigated and the three aspect ratios. For $\chi=3$ and 6 , the amplitude data points gather on a single curve for all $S$, which is the amplitude curve also obtained when $S=0$ and $\operatorname{Re}$ varies. The confinement induces a decrease of $R e$ and, for $\chi=3$ and 6 , the amplitudes of the oscillations of displacement and inclination are identical to those observed at the same $R e$ in an unbounded liquid. In particular, when the Reynolds number decreases beyond the threshold of path instability in the unbounded case $\operatorname{Re}_{c}(\chi, S=0)$, path oscillations disappear. Thin bodies $(\chi=10)$ exhibit a markedly different behaviour. The dispersed data points indicate that the amplitude is not dependent on $\operatorname{Re}$ alone. As mentioned previously, the increase of the confinement leads in this case to a decrease of the Reynolds number and an increase of the amplitude of oscillations, so that for the same $R e$, larger oscillations might be observed in a confined geometry than in the unbounded case. This behaviour might be related to the strong decrease for $\chi=10$ of the threshold of path instability $R e_{c}$ with the confinement ratio (figure 2). If we now plot the evolution of the amplitude of the oscillations as a function of the relative distance to the threshold of path instability, satisfactory clustering of the amplitudes for all $S$ on a unique curve is achieved (figure 14). Note that this has almost no impact on the curves for $\chi=3$ and 6 since for these aspect ratios $R e_{c}$ depends only weakly on $S$. These results indicate that, in both the confined and unconfined cases, the amplitude of the oscillatory motion is governed at leading order by the strength of the mean vertical motion and its distance relative to the threshold of path oscillations.

\subsection{Phase difference between the oscillations of velocity and inclination}

Figure 15 shows the phase difference between the oscillations of horizontal velocity and those of the inclination of a body as a function of the confinement ratio $S$, for three bodies with aspect ratios $\chi=3,6$ and 10. The behaviour for other Archimedes numbers is similar. For thinner bodies $(\chi=6$ and 10), the phase difference depends only weakly on $S$ and is larger than $90^{\circ}$, so that the bodies tend to slide along their path (Fernandes et al. 2005). For thick bodies $(\chi=3)$, the phase difference depends only weakly on the confinement ratio for $S \leqslant 0.4$ but increases sharply with $S$ for stronger confinement ratios. This is at variance with the behaviour in the unconfined case, where the phase difference decreases slowly when the Reynolds number is 
(a)

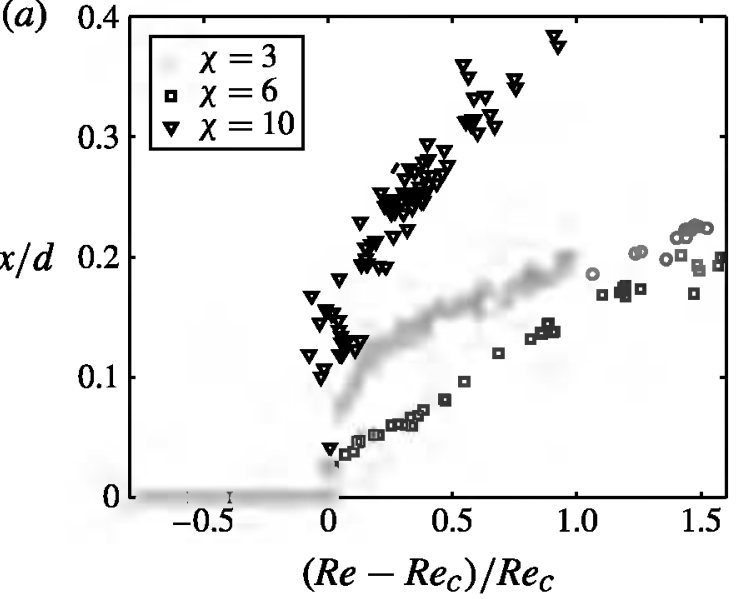

(b)

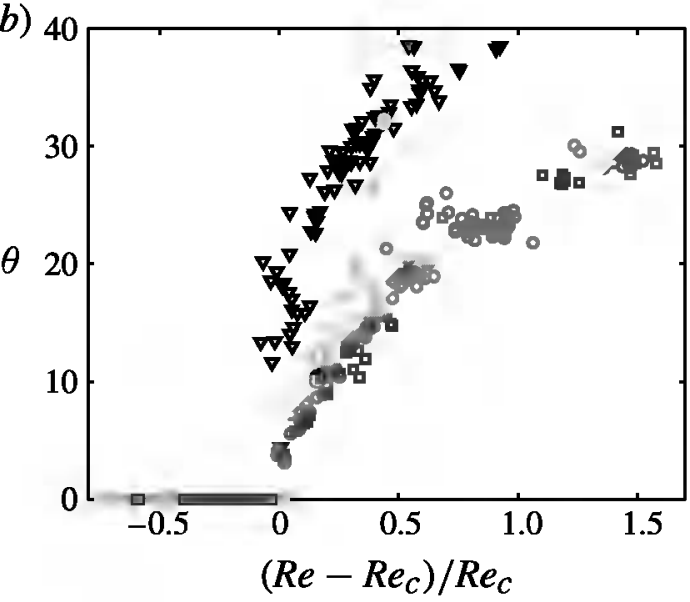

Figure 14. Amplitude of the oscillations as a function of the relative distance to the threshold of path instability, $\left(R e-R e_{c}\right) / R e_{c}$, for various confinement ratios: (a) horizontal position normalized with the body diameter; $(b)$ inclination $(\chi=3,6$ and 10$)$.

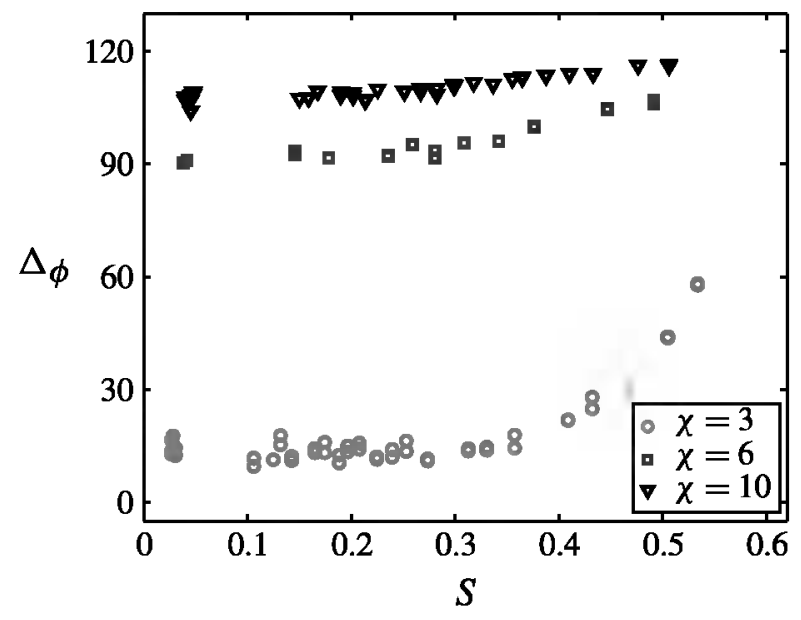

FIGURE 15. Phase difference between the oscillations of the horizontal velocity and those of the body inclination as a function of the confinement ratio $S$, for $\chi=3, \operatorname{Re}_{0}=255$, $d=10.5 \mathrm{~mm}, U_{m}(S=0)=24.9 \mathrm{~mm} \mathrm{~s}^{-1} ; \chi=6, R_{0}=285, d=15 \mathrm{~mm}, U_{m}(S=0)=$ $19.3 \mathrm{~mm} \mathrm{~s}^{-1} ; \chi=10, R_{0}=295, d=18 \mathrm{~mm}, U_{m}(S=0)=16.7 \mathrm{~mm} \mathrm{~s}^{-1}$.

decreased. The evolution of the phase difference for $\chi=3$ indicates that the body changes the way it evolves along its path: for weak confinements, the body tends to align its axis of symmetry with its velocity, while the body axis is increasingly delayed as the confinement increases.

\section{Discussion and conclusion}

This work was devoted to the free fall of disks in tubes having different diameters. Three-dimensional trajectography was used to record the body motion. We investigated the horizontal migration of the body, the nature of the path and its characteristics, in particular the mean velocity of fall and the oscillations in position and inclination of the body for the periodic paths. To obtain complementary quantitative information on the liquid motion, numerical simulations were performed in the comparable situation of a body held fixed in a confined uniform incoming flow. 
In the range of parameters investigated $\left(\rho_{s} / \rho_{f} \approx 1,40<A r<140,80<\operatorname{Re}_{0}<320\right.$ and $S<0.8$ ), we identified two types of motion, rectilinear and oscillatory. The latter takes the form of slightly helical paths, whose eccentricity $e$ depends only on the Reynolds number and is maximum just above the onset, $R \boldsymbol{e}_{c}$, of the path oscillations and then decreases as $R e$ increases. In the majority of the confined cases, the values of the eccentricity correspond to those observed experimentally in an unbounded medium (Fernandes et al. 2007), while the numerical simulations of Auguste (2010) provided in the latter case purely two-dimensional paths of nearly the same amplitudes and frequencies. It can therefore be expected that in an ideal situation (perfectly homogeneous body and liquid, no disturbances in the liquid, no geometrical defect of the body) purely two-dimensional zigzag motions would also be observed in this range of parameters. However, for $\chi=3$ and 6 , some helical motions of large amplitude were also observed for large $R e$ and large $S$. The onset of the periodic motion occurs above a critical Reynolds number $R e_{c}$, for all the confinement ratios. For bodies with aspect ratios $\chi=3$ and $6, R e_{c}$ depends only weakly on $S$, whereas for $\chi=10$ it decreases when $S$ is increased beyond $S \approx 0.2$ (by a factor of 2 for $S \approx 0.55$ relative to the unconfined situation). We compared the threshold of path instability to the thresholds of wake instability past the same bodies held fixed in a uniform confined flow. For thick bodies $(\chi=3)$, path instability occurs experimentally at slightly lower Reynolds number $(\sim 10 \%)$ than the first bifurcation, $R e_{c 1}$, corresponding to the loss of axial symmetry in the wake of the fixed body, as also occurs in the unbounded case. For thin bodies $(\chi=10)$, however, path instability leading to large-amplitude oscillations of the body is observed for weak confinement $(S \leqslant 0.2)$ only for Reynolds numbers much larger than $R e_{c 1}$ and even $R e_{c 2}$, which corresponds to the appearance of unsteadiness in the wake of the fixed body. For thin bodies, $R e_{c}$ then decreases sharply with $S$ while $R e_{c 1}$ and $R e_{c 2}$ evolve only weakly with $S$, so that the difference between these thresholds decreases until it vanishes around $S \approx 0.4$, after which $R e_{c}$ is smaller than $R e_{c 1}$. However, it might be expected that, in an ideal configuration for $R e$ in the range $\left[R e_{c 1}, R e_{c}\right]$, the thin body would not follow a vertical straight path but might oscillate irregularly with very low amplitudes, since this is known to occur in the absence of confinement (Auguste 2010).

We observed that no significant migration of the bodies in the horizontal plane occurred for $S \leqslant 0.4$, while for stronger confinement ratios the bodies migrate towards the centre of the tube during their fall, for any type of path. For given Archimedes number $A r$ and aspect ratio of the body $\chi$, the mean vertical velocity of the body and the Reynolds number $R e$ decrease when the confinement ratio $S$ increases. To gain insight into this behaviour, we investigated how the attached wake about a fixed body in a uniform bounded flow is modified when $S$ is varied. Numerical simulations performed just before the wake destabilization at $R e=R e_{c 1}$ revealed that the length of the stable attached wake decreases with $S$, while the maximum velocity $V_{w}$ (measured on the axes of symmetry of the body and the tube) increases with $S$. We then introduced a Reynolds number $R e^{*}$, which is proportional to $R e$ and whose dependence on $\chi$ and $S$ is given by empirical relations fitting the evolution of $V_{w}$ with the parameters $\chi$ and $S$. $R e^{*}$ may therefore be considered as a measure of how the intensity of the attached vortex in the wake of the body is modified relative to the incoming flow velocity or mean fall velocity when $\chi$ and $S$ vary. One of the most interesting results of this work is that for a freely moving body with given $A r$ and $\chi$, $R e^{*}$ remains constant when the confinement ratio $S$ changes, regardless of the nature of the path. This suggests that the mean vertical velocity (i.e. $R e$ ) adjusts accordingly and 
can therefore be predicted for any value of $S$, once a single case is known, by means of the empirical relation proposed.

The effect of the confinement ratio on the characteristics of the periodic motion was investigated in detail for the three aspect ratios. We have shown that, for a given Archimedes number, thick bodies $(\chi=3)$ tend to stabilize and to go back to a rectilinear path when the confinement ratio increases, while thin bodies $(\chi=10)$ display oscillations of growing amplitude with $S$ until they touch the tube (at about $S=0.5$ ). Remarkably, for a given aspect ratio, the amplitudes of the oscillations of displacement and inclination gather on a single curve for all the confinement ratios $S$ (including $S=0$ ), which depends only on the relative distance of the Reynolds number to the threshold of path instability.

\section{Acknowledgements}

The authors warmly thank F. Auguste, D. Fabre and J. Magnaudet for providing the numerical code and a stimulating working environment. They are also grateful to G. Ehses, H. Ayroles and S. Cazin for the technical support provided for the experiments.

\section{REFERENCES}

Auguste, F. 2010 Instabilités de sillage générées derrière un corps solide cylindrique, fixe ou mobile dans un fluide visqueux. PhD thesis, Université Paul Sabatier, Toulouse, France: http://thesesups.ups-tlse.fr/1186/.

Auguste, F., Fabre, D. \& Magnaudet, J. 2010 Bifurcations in the wake of a thick circular disk. Theor. Comput. Fluid Dyn. 24, 305-313.

BhATTACHARYYA, S. \& MAITI, D. 2006 Vortex shedding suppression for laminar flow past a square cylinder near a plane wall: a two-dimensional analysis. Acta Mechanica 184, 15-31.

CAMARRI, S. \& GianNETTI, F. 2007 On the inversion of the von Kármán street in the wake of a confined square cylinder. J. Fluid Mech. 574, 169-178.

CAMARri, S. \& GiannetTI, F. 2010 Effect of confinement on three-dimensional stability in the wake of a circular cylinder. J. Fluid Mech. 642, 477-487.

Chen, J. H., Pritchard, W. G. \& Tavener, S. J. 1995 Bifurcation for flow past a cylinder between parallel planes. J. Fluid Mech. 284, 23-41.

ChHabra, R. P., Agarwal, S. \& Chaudhary, K. 2003 A note on wall effect on the terminal falling velocity of a sphere in quiescent Newtonian media in cylindrical tubes. Powder Technol. 129 (1-3), 53-58.

Cliffe, K. A., SPEnCe, A. \& TAvener, S. J. 2000 O(2)-symmetry breaking bifurcation: with application to the flow past a sphere in a pipe. Intl J. Numer. Meth. Fluids 32 (2), 175-200.

Clift, R., Grace, J. \& Weber, M. E. 1978 Bubbles, Drops and Particles. Academic Press.

DELOZE, T. 2011 Couplage fluide-solide appliqué à l'étude de mouvement d'une sphère libre dans un tube vertical. PhD thesis, Université Louis Pasteur, Strasbourg, France.

ERN, P., Fernandes, P. C., Risso, F. \& MAgnaudet, J. 2007 Evolution of wake structure and wake-induced loads along the path of freely rising axisymmetric bodies. Phys. Fluids 19 (11), 113302.

ERn, P., Risso, F., FABRE, D. \& MAGNAUDET, J. 2012 Wake-induced oscillatory paths of freely rising or falling bodies. Annu. Rev. Fluid Mech. 44, 97-121.

FENG, J., HU, H. H. \& JOSEPH, D. D. 1994 Direct simulation of initial value problems for the motion of solid bodies in a Newtonian fluid. Part 1. Sedimentation. J. Fluid Mech. 261, 95-134.

Fernandes, P. C., ERN, P., Risso, F. \& Magnaudet, J. 2005 On the zigzag dynamics of freely moving axisymmetric bodies. Phys. Fluids 17 (9), 098107.

Fernandes, P. C., Risso, F., ERn, P. \& Magnaudet, J. 2007 Oscillatory motion and wake instability of freely rising axisymmetric bodies. J. Fluid Mech. 573, 479-502. 
Figueroa-Espinoza, B., Zenit, R. \& LEgEndRe, D. 2008 The effect of confinement on the motion of a single clean bubble. J. Fluid Mech. 616, 476-480.

KIM, I., Elghobashi, S. \& SiRignano, W. A. 1993 Three-dimensional flow over two spheres placed side by side. J. Fluid Mech. 246, 465-488.

LEGENDRE, D. \& MAGNAUdET, J. 1998 The lift force on a spherical bubble in a viscous linear shear flow. J. Fluid Mech. 368, 81-126.

Legendre, D., Magnaudet, J. \& Mougin, G. 2003 Hydrodynamic interactions between two spherical bubbles rising side by side in a viscous liquid. J. Fluid Mech. 497, 133-166.

Maheshwari, A., ChHABRA, R. P. \& Biswas, G. 2006 Effect of blockage on drag and heat transfer from a single sphere and an in-line array of three spheres. Powder Technol. 168 (2), $74-83$.

SAHIN, M. \& OWENS, R. G. 2004 A numerical investigation of wall effects up to high blockage ratios on two-dimensional flow past a confined circular cylinder. Phys. Fluids 16 (5), 1305-1320.

TAKEMURA, F. \& MAGNAUdeT, J. 2003 The transverse force on clean and contaminated bubbles rising near a vertical wall at moderate Reynolds number. J. Fluid Mech. 495, 235-253.

TAVener, S. J. 1994 Stability of the O(2)-symmetric flow past a sphere in a pipe. Phys. Fluids 6 (12), 3884-3892.

Wham, R. M., Basaran, O. A. \& BYERS, C. H. 1996 Wall effects on flow past solid spheres at finite Reynolds number. Ind. Engng Chem. Res. 35 (3), 864-874.

Yu, Z., Phan-Thien, N. \& TANner, R. I. 2004 Dynamic simulation of sphere motion in a vertical tube. J. Fluid Mech. 518, 61-93.

Zovatto, L. \& PEDRIZZETTI, G. 2001 Flow about a circular cylinder between parallel walls. J. Fluid Mech. 440, 1-25. 